Revue d'histoire de l'Amérique française

AH REVUE D.HISTOIRE DE L'AMÉRIQUE FRANÇAISE

\title{
Le développement des disparités régionales en Gaspésie
}

1760-1960

\section{Maryse Grandbois}

Volume 36, numéro 4, mars 1983

URI : https://id.erudit.org/iderudit/304092ar

DOI : https://doi.org/10.7202/304092ar

Aller au sommaire du numéro

Éditeur(s)

Institut d'histoire de l'Amérique française

ISSN

0035-2357 (imprimé)

1492-1383 (numérique)

Découvrir la revue

Citer cet article

Grandbois, M. (1983). Le développement des disparités régionales en Gaspésie 1760-1960. Revue d'histoire de l'Amérique française, 36(4), 483-506.

https://doi.org/10.7202/304092ar d'utilisation que vous pouvez consulter en ligne.

https://apropos.erudit.org/fr/usagers/politique-dutilisation/ 


\section{LE DÉVELOPPEMENT DES DISPARITÉS RÉGIONALES EN GASPÉSIE 1760-1960}

MARYSE GRANDBOIS

Département des sciences juridiques Université du Québec à Montréal

Le début des années soixante marque pour les gouvernements fédéral et québécois l'instauration d'un programme d'aménagement du territoire en Gaspésie. Il s'agit d'un ensemble de législations québécoises et canadiennes, destinées à réduire les disparités régionales et à favoriser le développement économique. Ce programme consiste d'abord en une vaste étude menée par le Bureau d'aménagement de l'Est du Québec ${ }^{1}$, bientôt suivie de la conclusion d'ententes fédérales-provinciales de développement ${ }^{2}$. L'aménagement de la Gaspésie s'étendra sur vingt ans. Les gouvernements y mèneront une complète restructuration spatiale, une véritable reterritorialisation: création de deux villes, en lieu et place des villages de la péninsule ${ }^{3}$, fermeture des villages des terres intérieures et relocalisation des habitants ${ }^{4}$, création d'un parc national ${ }^{5}$, aménagement d'infrastructures favorisant le développement du tourisme et des pêcheries. Le droit fait alors en Gaspésie une entrée fracassante.

À cette époque, en 1960, la Gaspésie présente de multiples disparités, de population, de revenu, de développement des ressources, qui en font une région marginale et isolée. Selon les témoigna-

1 Bureau d'aménagement de l'Est du Québec, Plan de développement de la régionpilote: Bas Saint-Laurent, Gaspésie et les Îles-de-la-Madeleine, 10 cahiers et 30 annexes techniques (Québec, 1966).

2 Entente générale de Coopération Canada-Québec, 1968-1976, Le plan de développement du Bas Saint-Laurent, de la Gaspésie et des Îles-de-la-Madeleine, conclue le 26 mai 1968, amendée le 10 septembre 1971, Ministère de l'expansion économique régional Office de planification et de développement du Québec (éditeur officiel, Ottawa, 1972). (Désormais MEER-OPDQ).

3 Les villes de Gaspé, Lois du Québec 1970, chap. 76, et de Percé, Lois du Québec 1970, chap. 77.

4 Ministère des affaires sociales, a.c. 2874-72 et a.c. 1452-74, Programme d'aide à la migration, non publié.

5 Création du parc Forillon, en vertu de la loi sur les parcs nationaux, Statuts révisés du Canada, 1970, chap. N-13. Voir aussi Loi concernant le parc Forillon et ses environs, Lois du Québec 1970, chap. 32. 
ges entendus devant la Commission d'enquête Tremblay, quelques années plus tôt (1954-1956) ${ }^{6}$, l'agriculture périclite, les forêts sont pillées, la pêche fortement subventionnée et le tourisme négligé ${ }^{7}$. En guise de solution, les gouvernements proposent une restructuration spatiale et un réaménagement «rationnel». La Gaspésie, décident-ils, doit se moderniser et s'intégrer à un nouvel ensemble territorial. Cette solution s'impose, comme si les gouvernements venaient de découvrir, à la faveur des audiences de la Commission Tremblay, la situation désespérante des pêcheurs et habitants gaspésiens.

Pourtant l'État entretient des rapports étroits avec cette région depuis longtemps déjà. La Gaspésie doit même son développement et son peuplement à cette dépendance continuelle et croissante, obtenue par une législation d'exception. Les disparités régionales en 1960 datent en fait de deux siècles. Elles ont été créées, entretenues et reproduites de toutes pièces par l'État, qui y produit un droit parallèle depuis deux cents ans. De l'histoire juridique de ce développement, nous pouvons dégager une double constante: d'abord, la péninsule se développe dans un isolement géographique voisin de l'abandon; ensuite, dans une relation étroite de dépendance à l'État.

Comme région québécoise, la Gaspésie a connu différentes territorialisations depuis le Régime français: avec le Traité de Paris en $1763^{8}$, l'Acte de Québec en $1774^{\circ}$, l'Acte d'Union en $1840^{10}$, l'Acte de l'Amérique du Nord britannique en $1867^{11}$. Chaque fois, ces lois constitutionnelles ont signifié une nouvelle articulation régionale et, pour la Gaspésie particulièrement, de nouvelles législations et une dépendance accrue.

L'existence administrative et juridique de la Gaspésie remonte au Régime français. Les premiers établissements permanents ne

\footnotetext{
Statuts du Québec, 1953, chap. 4.

G.-E. Blanchard, La Gaspésie, ordonnance de ses valeurs et problèmes constitutionnels, mémoire soumis à la Commission royale d'enquête présidée par le juge Thomas Tremblay (Chandler, 1954). Voir aussi OPDQ, L'Est du Québec, d'hier à aujourd'hui (Québec, 1980).

8 Traité de Paris, 10 février 1763. Sir Arthur George Doughty et Adam Shortt, Documents relatifs à l'histoire constitutionnelle du Canada, 1759-1791, Archives publiques, Bureau de publication des documents historiques (Ottawa, Imprimeur du Roi, 1921), 21 . ments..., 552 .

9 Acte de Québec, 22 juin 1774, 14 GEO III chap. 83, Doughty et Shortt, Docu-

10 Acte d'Union, 23 juillet 1840, 3 et 4 Vict. chap. 35, Maurice Ollivier, Acte de l'Amérique du Nord britannique et Statuts connexes (Ottawa, Imprimeur de la Reine, 1962), 21.

11 Acte de l'Amérique du Nord britannique, 1867, 30-31 Vict. chap. 3. Maurice Ollivier, Acte de l'Amérique du Nord britannique,... 65.
} 
s'édifient qu'au milieu du XVIIe siècle, sous l'administration des compagnies (1628-1674) ${ }^{12}$. Les rares concessions de terres sont motivées par la pêche, le seigneur obtenant en titre l'exclusivité des droits sur toute la côte, en contrepartie de quoi il s'engage à établir des colonies de peuplement. Cet engagement devient contrôlé en 1663, avec la création du Conseil souverain: les concessions non défrichées peuvent alors être révoquées ${ }^{13}$. Après 1675, sous le gouvernement de la colonie (1675-1760) ${ }^{14}$, la Gaspésie se peuple davantage. C'est encore la pêche qui motive l'acquisition des seigneuries. Les droits des seigneurs demeurent exclusifs et sont protégés par la justice des intendants qui prononcent des jugements contre les contrevenants ${ }^{15}$. À la fin du Régime français, la guerre trouble grandement la pêche au large des côtes gaspésiennes. Mais la métropole ne fortifie pas la côte, tout au plus arme-t-elle quelques bateaux, suite aux attaques anglaises ${ }^{16}$, pour que la pêche se poursuive. Après la Conquête, sous la domination anglaise, la Gaspésie devient plus qu'un comptoir de pêche. La région se développe toutefois comme une féodalité, isolée et greffée au territoire. Cette situation de marginalité ne se résorbera pas avec les années. Des inégalités de développement se feront sentir fortement dès le XIXe siècle.

Dans cet article, nous constaterons la mise en place de cette situation de dépendance et de disparités régionales, mise en place où l'État et le droit jouent un rôle de premier plan. Il s'agit bien de droit de l'aménagement du territoire, l'État intervenant pour créer en Gaspésie des conditions de croissance bien particulières. Dans la première partie, nous retracerons l'histoire juridique de ces disparités pendant la période $1760-1840$, avant la promulgation de

12 De 1628 à 1663, la Compagnie des Cent-Associés détient toutes les terres de la Nouvelle-France, à l'exception des terres concédées à Louis Hébert (1623) et aux Jésuites (1626), en vertu de pouvoirs donnés par le Roi. Voir «Acte pour l'établissement de la Compagnie des Cent-Associés, 29 avril $1627 »$, dans Édits, ordonnances royaux, Déclarations et arrêts du Conseil d'État du roi concernant le Canada (Québec, Fréchette, 1854), 5. En 1664, la Compagnie des Indes occidentales remplace la Compagnie des Cent-Associés comme administrateur des terres. Voir «Établissement de la Compagnie des Indes occidentales», Édits, ordonnances ..., 40. Cette période prend fin en 1674.

13 Ordonnance du 21 mars 1663. «Révocation des concessions non défrichées», Édits, ordonnances ..., 33.

14 «Déclaration du roi qui confirme et règle l’établissement du Conseil souverain du Canada, 5 juin 1675», Édits et ordonnances ..., 83.

15 «Jugement qui déboute les sieurs Marcot et Chastenay de la prétention qu'ils ont de s'arroger le droit de pêche sur la devanture de leurs terres, réservé par le sieur Robineau, seigneur de Portneuf», 25 juillet 1723. Complément des ordonnances et Jugements des Intendants du Canada (Québec, Fréchette, 1856), 203. "Ordonnance qui fait défense à toutes personnes de troubler le sieur Sarrazin et ses associés dans sa seigneurie, tant dans l'exploitation d'une carrière d'ardoise que dans ses pêcheries de morue», 14 octobre 1729, Complément des ordonnances..., 456.

16 David Lee, «Les Français en Gaspésie 1534-1760», dans Lieux historiques canadiens, Cahiers d'archéologie et d'histoire, 3 (1972): 39. 
l'Acte d'Union. La deuxième partie portera sur les années 18401960 , période où ces disparités s'accentuent et se reproduisent, malgré l'amélioration générale des communications, période où se constitue un véritable droit d'exception.

\section{1 - Naissance et croissance d'une inégalité: 1760-1840}

Aucune mesure de la métropole française n'encourage l'établissement des colons en région éloignée. Aussi les habitants sontils peu nombreux à s'établir en Gaspésie. Au moment de la Conquête, en 1760 , la région compte 18 seigneuries ${ }^{17}$ et 150 à 300 habitants dispersés ${ }^{18}$. En 1765 , le recensement dénombrera 318 Gaspésiens: 109 à Gaspé, 209 à la baie des Chaleurs ${ }^{19}$.

Au moment de la Conquête, le Québec connaît les mêmes institutions que la France. Les institutions juridiques françaises n'ont subi que des modifications mineures en Nouvelle-France ${ }^{20}$. Les colons-censitaires se trouvent d'ailleurs peu concernés par le droit, si l'on excepte la justice pénale. En cette matière, les colons canadiens sont soumis à la Grande Ordonnance criminelle du Roi de France du mois d'août 1670; même si elle n'a jamais été enregistrée en Nouvelle-France, l'Ordonnance de $1670 \mathrm{y}$ est suivie à la lettre ${ }^{21}$. La justice du Roi semble cependant s'être légèrement adoucie en traversant l'Atlantique: en Nouvelle-France, la torture au moment de l'interrogatoire s'utilise peu et l'acquittement des prévenus se rencontre plus fréquemment ${ }^{22}$.

Les capitulations de Québec et de Montréal en 1760 accordent aux habitants de la colonie des privilèges limités, au nombre desquels on compte le libre exercice de leur religion et un régime militaire de tolérance ${ }^{23}$. Trois ans plus tard, le Traité de Paris confirme la liberté de pratique de la religion catholique et les droits des habi-

17 Jean Bouffard, Traité du domaine (Québec, Le Soleil, 1921), appendice D: Extrait des Titres seigneuriaux, 147-218.

${ }_{18} \mathrm{Ce}$ sont les chiffres les plus souvent cités. Selon une étude récente, il aurait cependant compté «500 à 600 colons établis dans la péninsule à la veille de la conquête anglaise.» Voir Jules Bélanger, Marc Desjardins et Yves Frenette, Histoire de la Gaspésie (Montréal, Boréal Express, 1981), 117.

19 Recensements du Canada, 1665 à 1871 (Ottawa, 1876), 4:65. bec, PUL, 1978), 5 ss.

20 André Lachance, La justice criminelle du roi au Canada au XVIIIe siècle (Qué-

21 B.-A. Testard de Montigny, Histoire du droit canadien (Montréal, Eusèbe Sénécal, 1869), 30 et 195.

22 André Lachance, Le procès criminel en Canada au XVIIIe siècle (1712-1748) (1974), 5, Revue de droit de l'Université de Sherbrooke, 105-108.

${ }_{23}$ Capitulation de Québec, 18 septembre 1759 et Capitulation de Montréal, 8 septembre 1760. Thomas Chapais, Cours d'histoire du Canada (Québec, Garneau, 1919), I: appendice I et II: 249-268, Doughty et Shortt, Documents ... précité, 1-23. 
tants sur leurs biens. En 1763 toujours, la Proclamation royale remplace par la Loi anglaise la Coutume de Paris et crée des tribunaux où l'anglais doit être la seule langue officielle ${ }^{24}$.

Les Canadiens tiennent ardemment aux lois françaises en matière civile. Ils acceptent cependant les lois criminelles anglaises, dont la douceur contraste avec la rigidité et l'incertitude du droit français ${ }^{25}$. Ces Canadiens doivent patienter jusqu'en 1774 pour retrouver à peu près les frontières de l'ancienne Nouvelle-France et les lois civiles françaises. Cette année-là, après de longues enquêtes et à la veille de l'Indépendance américaine, Londres adopte l'Acte de Québec ${ }^{26}$. Par cet acte, les Canadiens obtiennent la liberté religieuse: on reconnaît officiellement la dîme et on abolit le serment du test. L'Acte de Québec accorde même une participation au Conseil de la colonie. Ce rétablissement des droits des colons canadiens se poursuit ensuite avec l'Acte constitutionnel de 1791 ${ }^{27}$, lequel sépare le territoire en Haut et Bas-Canada, accordant une Assemblée législative aux Canadiens.

Après l'Acte de Québec, en 1774, les Canadiens français conservent le régime seigneurial, mais les terres se découpent dorénavant selon la tenure anglaise du franc et commun soccage. Le territoire se divise en cantons d'étendue variable. De 1763 à 1840, les cantons seront créés sous l'autorité impériale, par proclamation dans la Gazette officielle ${ }^{28}$. Dans ces cantons, les terres sont attribuées aux colons moyennant certaines conditions d'établissement: défricher, bâtir, exploiter. Quand les conditions sont accomplies, le colon reçoit des lettres patentes et devient propriétaire de cette terre.

Pour les colons de la Gaspésie, la Conquête amorce un double isolement. Ils sont d'abord mis en minorité numérique, du fait de l'arrivée de Loyalistes américains en très grand nombre: 200 familles ${ }^{29}$. Ces familles reçoivent l'aide de l'État, sous forme de l'octroi d'une terre et de vivres pendant trois ans. La Gaspésie prend alors figure de terre de refuge pour les Acadiens et les Loyalistes américains.

\footnotetext{
${ }^{24}$ La proclamation royale du 7 octobre 1763, Thomas Chapais, Cours d'histoire $d u$ Canada, appendice III: 269-273; Doughty et Shortt, Documents..., 124.

${ }_{25}$ Edmond Lareau, Histoire du droit canadien, Tome I, Domination française (Montréal, Librairie générale de droit et de jurisprudence, 1888): 307.

${ }_{26}$ Acte de Québec, 22 juin 1774, Thomas Chapais, Cours d'histoire du Canada, appendice IX, 325-333. Doughty et Shortt, Documents..., 552.

27 Acte constitutionnel de 1791, Doughty et Shortt, Documents ..., 1013.

28 Jean Bouffard, Traité du domaine, précité, 16.

29 Jules Bélanger, Marc Desjardins et Yves Frenette, Histoire de la Gaspésie, préci-
} té: 144 . 
Avec l'installation de pêcheurs des îles anglo-normandes Jersey et Guernesey, les Francophones ne forment plus qu'un des groupes gaspésiens, dans la diversité ethnique culturelle et religieuse de la péninsule. De plus, à cette époque, le voyage de Gaspé à Québec prend quinze jours, parfois vingt. C'est dire l'isolement de ce groupe, coupé en même temps des relations avec les autres Francophones.

Les seigneuries gaspésiennes du Régime français sont toutes abandonnées; elles passent à des militaires, des marchands ou des fonctionnaires anglais ${ }^{30}$. Le recensement de 1765 dénombre 318 Gaspésiens. En 1777 leur nombre atteint 625; au début du XIXe siècle, ils seront $5000^{31}$. La population se concentre à la baie des Chaleurs. En 1788 le gouvernement forme un nouveau district judiciaire: le district de Gaspé ${ }^{32}$. Gaspé acquiert ainsi une existence administrative comme unité territoriale ${ }^{33}$. Ce territoire se greffera cependant au Québec méridional comme un sous-ensemble isolé. La population se trouve même écartée des questions politiques nationales, ces questions étant prises en charge par les quelques notables régionaux. Ces structures d'isolement et de dépendance permettront l'instauration de nouveaux rapports féodaux. La Gaspésie peut ainsi devenir le fief d'une famille jersiaise adonnée au commerce de la morue: les Robin.

\section{A - Le monopole de Charles Robin}

Aussitôt après la Conquête, des bateaux anglais viennent pêcher dans le golfe Saint-Laurent. La concurrence est vive. Des entreprises de Québec, d'Halifax, d'Angleterre et des États-Unis acquièrent des intérêts dans la région, de même que des marchands venus des îles anglo-normandes, Jersey et Guernesey. La pêche continue d'être saisonnière. Charles Robin, originaire de l'île Jersey, forme en 1764 une compagnie: Robin, Pipon et Co. et vient en éclaireur visiter la Gaspésie ${ }^{34}$. L'année suivante, il ouvre un comptoir de pêche permanent à Paspébiac, dans la baie des Chaleurs. C'est là le premier établissement permanent pour la pêche en Gaspésie. Pour sa contribution à la sédentarisation de la pêche, Charles Robin se voit la tâche facilitée par le gouvernement anglais. Pour les autorités coloniales, c'est l'occasion de peupler un peu la

\footnotetext{
${ }^{30}$ Ibid., 163.

31 Recensements du Canada 1665-1871, précité, 83.

32 Acte concernant la division du Bas-Canada en district pour l'administration de la justice, Statuts refondus du Bas-Canada, (1861), chap. LXXV: 644.

${ }_{33}$ Divisions territoriales pour des fins générales, Statuts refondus du Bas-Canada, (1861), chap. LXXV: 627.

${ }_{34}$ John $P$. Le Garignon, «Une étude socio-historique de la présence jersiaise en Gaspésie», dans Revue d'histoire de la Gaspésie, XVI, 2-3 (avril-septembre 1978): 60.
} 
région. L'État accorde donc à Charles Robin des conditions commerciales qui, alliées à une exploitation systématisée, lui permettront de supplanter tous ses concurrents. Avec l'aide de l'État, ce marchand édifiera en Gaspésie un empire comparable à celui de la Compagnie de la baie d'Hudson.

À cette époque, selon les dispositions de l'Acte de navigation de 1764 du Parlement britannique, tous les navires en partance pour Terre-Neuve et la Nouvelle-Angleterre doivent partir d'un port britannique. Cette loi oblige donc les capitaines et armateurs jersiais à faire escale dans un port anglais avant de naviguer vers le Canada. Peu s'y conforment. Deux navires de Charles Robin, en contravention avec ces dispositions, sont saisis par les autorités anglaises. Les marchands jersiais obtiennent en 1768 l'amendement de l'Acte de navigation ${ }^{35}$. Trois ans plus tard le Parlement britannique dédommage Charles Robin pour les frais occasionnés par les saisies. En 1773, le gouvernement anglais accepte de nouveau d'indemniser ce marchand pour des dommages de 6000 livres, encourus à la suite de démêlés avec des corsaires américains ${ }^{36}$.

Charles Robin se retrouve alors parmi les rares marchands de poisson de la péninsule. La guerre d'Indépendance américaine (1776-1783) appauvrit ses concurrents, quand ils ne font pas faillite $^{37}$. La Loi des pêcheries de $1788^{38}$ permet à la compagnie Robin de prendre plus d'expansion. Cette loi régularise la pêche au profit des marchands britanniques et jersiais. Selon les termes de cet acte, tout capitaine de vaisseau peut établir une pêche sédentaire partout où le territoire n'est pas concédé, et peut construire et s'approprier les berges avoisinantes ${ }^{39}$. Le gouvernement anglais ouvre ainsi la Gaspésie à la libre initiative des marchands. La péninsule deviendra un relais du capital marchand anglais et jersiais ${ }^{40}$, une assise de ce capital, façonnée par ses exigences.

Dès lors, Charles Robin monopolise les terres du littoral et multiplie ses magasins. Il organise bientôt un système économique complet, fondé sur l'établissement d'un double monopole ${ }^{41}$. Au

35 Arthur G. Le Gros, «Charles Robin on the Gaspé Coast 1766», dans Revue d'histoire de la Gaspésie, 111, 3 (1965): 148-155.

36 John P. Le Garignon, «Une étude socio-historique...» précité, 66.

37 Jules Bélanger et al, Histoire de la Gaspésie, précité: 209.

38 Acte qui règle les pêches dans le fleuve Saint-Laurent, les baies de Gaspé et des Chaleurs, à l'île de Bonaventure et sur le rivage vis-à-vis Percé, 28 GEO. III, chap. 6, Statuts du Québec, 1774-1792 (Québec, Imprimeur du Roi, 1797), 154 ss.

39 Ibid., art. 2 et 3.

40 Roch Samson, «Gaspé 1760-1830, L’action du capital marchand chez les pêcheurs» dans Anthropologie et sociétés, 5 (1981): 57-85. Gaspésie: 68.

${ }^{41}$ John P. Le Garignon, «Une étude socio-historique...», Revue d'histoire de la 
printemps, les pêcheurs doivent en effet s'approvisionner à crédit en agrès de pêche dans les magasins Robin, seul commerçant. Robin achète le poisson et paie les pêcheurs en fin de saison. Les dettes dépassent toujours le revenu, ce qui signifie que le pêcheur doit s'endetter davantage pour acheter ses provisions d'hiver et ses agrès de pêche au printemps suivant. Comme le pêcheur ne peut jamais rembourser toute sa dette, il s'inféode davantage d'une année à l'autre à la famille Robin. Le contrôle économique de Charles Robin s'étend à la production, au transport et à la consommation. Il établit le monopole de la vente du sel, de sorte que les pêcheurs doivent lui livrer tous leurs poissons ${ }^{42}$; il monopolise aussi le transport et les terres pour empêcher que les pêcheurs ne puissent s'adonner à l'agriculture. Il fournit les barques contre redevances et achète toute la production à prix ridiculement bas, payant pour moitié en argent, pour moitié en crédit dans ses magasins. Il organise même un trafic de main-d'oeuvre avec l'île Jersey, dont il est originaire. La Compagnie Robin possède ses propres navires qui font la navette Gaspésie-Jersey-Europe, rapportant tantôt le sel, tantôt la main-d'oeuvre et exportant le poisson. Le pêcheur gaspésien n'a d'alternative que de pêcher pour Robin ou mourir de faim. À la fin du XIXe siècle, 11000 pêcheurs et 4000 travailleurs du poisson soutiennent cet empire. Les installations Robin comprennent huit habitations, dix magasins, une réserve de sel, une voilerie, un atelier de garniture et onze hangars; la compagnie Robin exporte 30 à 50 tonnes d'huile de foie de morue, 100 barils de poisson mariné et 22000 à 25000 quintaux de morue sèche par an ${ }^{43}$.

À cette époque, l'isolement de la péninsule demeure total. La seule voie de communication s'avère le chemin privé, la loi sur les chemins de $1796^{44}$ imposant aux propriétaires riverains les frais de construction de ces routes. En 1808 , ces chemins se révélant très insuffisants, l'État prend en partie en charge les chemins et ponts de Gaspé 45 .

Quand le gouvernement se met à concéder des terres, Charles Robin, qui jouit d'un pouvoir absolu, convainc les pêcheurs qu'un lot de dix arpents leur suffit, pour ne pas les détourner de la

42 Gérard Guité, Le milieu gaspésien et ses ressources, mémoire, licence en sciences sociales (Québec, Université Laval, 1940), 31.

43 Jules Bélanger et al, Histoire de la Gaspésie, précité, 210.

44 Loi sur les chemins et ponts, 36 GEO III, 1796, Statuts refondus du BasCanada, I (Montréal, Imprimeur de la reine, 1862), Cédule C, 1046.

${ }_{45}$ Acte qui pourvoit plus efficacement à faire changer et entretenir les chemins et ponts dans le district inférieur de Gaspé, 48 GEO III, chap. 25. Actes et ordonnances révisés du Bas-Canada (Montréal, Imprimeur de la reine, 1845), 380. 
pêche ${ }^{46}$. Mais la compagnie Robin et Co. réclame et obtient en 1792 une concession de mille acres de terres, laquelle sert d'abord de réserve de bois; par la suite, la compagnie vend des parcelles aux pêcheurs ${ }^{47}$.

Tous les pêcheurs de la péninsule s'endettent auprès des entreprises Robin. Charles Robin se comporte comme un gouverneur de la Gaspésie. Riche, puissant et craint, il tente de maintenir les conditions féodales qui font sa fortune. Il établit un réseau d'alliances avec les représentants de l'autorité civile. Il empêche la fondation d'écoles lesquelles détourneraient les jeunes de la pêche. Les autorités coloniales reconnaissent en Charles Robin l'homme le plus influent en Gaspésie. Il reçoit en retour diverses gratifications: il siège comme commissaire au Conseil des terres de la Gaspésie, conseil qui accorde des terres aux nouveaux arrivants, et siège pendant une session comme juge à la Gaspé Court of Common Pleas $^{48}$. Il fait aussi ouvrir un bureau de douane à New Carlisle, près de ses installations ${ }^{49}$. Charles Robin se retirera en 1824, laissant à ses neveux la direction de ses affaires. La famille Robin conservera une grande emprise en Gaspésie, pendant un siècle mais elle connaîtra davantage de concurrence.

\section{$B$ - Les interventions gouvernementales}

Suite à de nombreuses plaintes des habitants de la péninsule, deux Commissions d'enquête sont créées par les autorités coloniales: la Commission des terres de Gaspé, en $1819^{50}$ et le Comité d'enquête de la Chambre d'Assemblée sur les pêcheries de Gaspé en $1823^{51}$.

La Commission des terres est due à l'initiative du député de Gaspé, James Cockburn. À cette époque, la plupart des Gaspésiens ne détiennent pas de titres de propriété; certains ont des billets de louage, d'autres rien du tout et plusieurs se disent propriétaires des mêmes terres. En l'absence de notaires dans le district, tous les actes officiels sont faits sous seing privé et la plus grande

\footnotetext{
46 Antoine Bernard, La Gaspésie au Soleil (Montréal, Clercs de Saint-Viateur, 1925), 184-185.

47 Thomas Pye, Images de la Gaspésie au XIXe siècle (1866), traduit et commenté par Jean Laliberté et André Lepage (Québec, Presse Comédix, 1980), 10, note 13.

48 David Lee, «La Gaspésie 1760-1867», dans Lieux historiques canadiens, Cahiers d'archéologie et d'histoire, 23 (1980): 157.

49 Jules Bélanger, Histoire de la Gaspésie, précité, 209.

50 Cette commission est formée par l'Acte pour assurer les habitants du district inférieur de Gaspé de la possession et jouissance de leurs terres, Statuts du Bas-Canada, 18151819, GEO III, 8: 259.

51 Ce comité de la Chambre d'Assemblée est formé après la première lecture de l'Acte pour mieux régler les pêches dans le district de Gaspé, et remet son rapport en décembre 1823. Journal de la Chambre d'Assemblée, 1823-1824, 33: 27.
} 
instabilité affecte les titres de propriété. La principale fonction de la Commission sera de reconnaître des titres dans des preuves écrites et de délimiter les propriétés ${ }^{52}$. La Commission reçoit aussi le mandat de s'enquérir des besoins de la Gaspésie en matière d'agriculture et de faire rapport sur la situation et les moyens d'améliorer la situation. Cette Commission réalise une vaste enquête: les commissaires entendent près de mille personnes et prennent connaissance de 631 recommandations. Robert Christie, secrétaire de la Commission, reçoit le support politique de Charles Robin ${ }^{53}$ qui veut profiter de son influence pour infléchir les politiques dans le sens de ses intérêts commerciaux. Robert Christie sera élu député de Gaspé en 1827. La Commission des terres remet son rapport final en 1825. Ses recommandations: encourager l'agriculture dans la péninsule, tracer des chemins et aménager le port de Gaspé.

L'influence de Charles Robin auprès de la Commission des terres lui sera utile à plus d'un titre. Â la même époque siège le Comité d'enquête de la Chambre d'Assemblée sur les pêcheries, comité formé pour arbitrer les différends entre les pêcheurs et les compagnies. Les griefs des pêcheurs portent sur le prix de la morue: des caboteurs pêchent dans le golfe et achètent la morue des Gaspésiens, payant jusqu'à quatre fois le prix offert par Charles Robin. Les griefs de la Compagnie concernent la pêche au hareng: les Gaspésiens gardent le hareng pour l'utiliser comme engrais sur leur terre, et la pêche à la morue en souffre car le hareng représente le meilleur appât pour la morue.

Suite à cette enquête, la Chambre d'Assemblée adopte l'Acte pour mieux régler les pêches dans le district inférieur de Gaspés4. Cette loi, qui confirme les droits des marchands sur les grèves ${ }^{55}$, interdit aux pêcheurs d'utiliser le capelan et le hareng comme engrais, ces poissons devant servir de boette pour la morue ${ }^{56}$. Une deuxième loi ne tarde pas à suivre: la Loi du dernier équipeur, en $1829^{57}$. Selon les dispositions de cette loi, les Gaspésiens doivent vendre leur poisson à la Compagnie qui leur fait crédit pour les agrès de pêche. Il devient donc impossible aux pêcheurs de vendre leurs prises au plus offrant s'ils s'endettent dans les magasins Robin. Comme les pêcheurs sont toujours endettés et liés à ces magasins, ils ne peuvent vendre leur poisson qu'aux entrepôts de la dice $\mathrm{E}$

Journal de la Chambre d'Assemblée 1823-24, appendice M, et 1824-25, appen-

3 Thomas Pye, Images de la Gaspésie..., 4.

54 Statuts provinciaux du Bas-Canada, II (1821-1824), GEO IV: 471.

55 Ibid.: art. 2.

56 Ibid.: art. 6.

57 Acte relatif aux pêches dans le comté de Gaspé, 14 mars 1829, 9 GEO IV, chap. 42, art. 10, Statuts du Bas-Canada (1829): 309. 
compagnie Robin. Cette dernière qui ne craint plus la concurrence des caboteurs du golfe, continuera d'acheter la morue à vil prix.

L'année suivante, en 1830 , les pêcheurs demandent le retrait de ces dispositions et l'abrogation d'une autre loi, prévoyant l'emprisonnement des matelots désertant les bateaux ${ }^{58}$. La requête dénonce aussi l'influence indue de la maison des Robin sur les électeurs et dénonce de nouveau l'instabilité des titres de propriété. La Chambre d'Assemblée forme un Comité d'enquête qui se rend en Gaspésie: il entend divers témoignages et fait rapport à la Chambre d'Assemblée ${ }^{59}$. Cette enquête permet de tracer un portrait de la situation du pêcheur gaspésien, mais elle n'est suivie d'aucune mesure gouvernementale.

Les Gaspésiens se plaignent à plusieurs reprises: le gouvernement néglige le district de Gaspé. L'État retire plus d'impôt dans la péninsule qu'il n'y dépense en services ${ }^{60}$. Le gouvernement n'administre aucun programme d'assistance sociale en Gaspésie, malgré la misère des habitants. Des pétitions fréquentes ne changent pas la situation. La seule aide gouvernementale consiste en cargaisons de farine, quand la famine menace ${ }^{61}$.

Dans la première moitié du XIXe siècle, les institutions gouvernementales concourent à renforcer l'isolement des Gaspésiens. Les fonctionnaires sont presque tous anglophones ( 33 sur 37 ) et les députés n'habitent pas la Gaspésie. Même si la population francophone représente plus de la moitié de la population totale, les électeurs sont presque tous anglophones. Un seul député francophone sera élu, peu avant la Confédération. Les seuls intérêts que présente la Gaspésie pour l'État demeurent la pêche et les Compagnies qui en assurent l'exploitation. Si l'État offre d'excellentes conditions commerciales aux compagnies de pêche, c'est parce que ces compagnies exercent un rôle d'autorité; elles remplacent l'État dans la région, garantissant à l'État la paix sociale.

Depuis la fin du XVIIIe siècle, notables et députés, demandent l'annexion de la Gaspésie au Nouveau-Brunswick. Londres qui ne voit pas là une revendication maritime, traduit cette demande comme un désir d'annexion au Canada anglais. En 1840, lors de l'adoption de l'Acte d'Union, la métropole propose de rat-

\footnotetext{
vol. 1 , chap. LVI: 495 .

58 Acte relatif à la désertion des matelots, Statuts refondus du Bas-Canada, 1862,

59 «Rapport du comité spécial sur la pétition de certains habitants du district de Gaspé se plaignant de divers griefs et autres références.» Journal de la Chambre d'Assemblée, 39 (1830), app. I., 19 mars 1980.

60 David Lee, "La Gaspésie 1760-1867», Lieux historiques canadiens, no 23 précité, 136-138.

61 Ibid., 134.
} 
tacher la Gaspésie au Haut-Canada, c'est-à-dire à la province anglaise. Mais ce rattachement est refusé par la population de la péninsule ${ }^{62}$. L'Acte d'Union divise alors la colonie canadienne en deux provinces: le Haut et le Bas-Canada, recouvrant à peu près les territoires de l'Ontario et du Québec actuel. Ce régime n'entraînera pas une autonomie régionale plus grande, mais bien au contraire, l'établissement d'une nouvelle dépendance à l'État qui se maintient intacte, pendant plus d'un siècle.

\section{2 - Le développement des disparités régionales en Gaspésie (1840-1960)}

La pêche à la morue connaît ses meilleures années pendant la période de 1840-1867. C'est aussi à cette époque que se constitue le capitalisme au Québec; celui-ci se développe d'emblée comme un capitalisme d'État, anglophone et nord-américain, en association continuelle avec l'État et les grands partis politiques canadiens, et dans l'ombre du capitalisme américain ${ }^{63}$. Le capitalisme maritime n'échappe pas à ce constat. Le gouvernement consolide la position des firmes jersiaises, dont il reconnaît la contribution importante au développement des pêcheries canadiennes. La concurrence se fait cependant plus forte. Des navires étrangers et de nouvelles compagnies pêchent dans le golfe Saint-Laurent.

La population de la péninsule atteint 7842 personnes en $1844^{64}$. L'isolement des Gaspésiens demeure aussi grand qu'au siècle précédent car les liaisons maritimes et terrestres ne s'améliorent pas. Les pêcheurs délaissent l'agriculture, devenue marginale à cause du faible rendement des terres. Avec le développement du commerce du blé, les marchands anglais achètent des seigneuries pour y construire des moulins à farine. Le régime seigneurial est bientôt définitivement aboli ${ }^{65}$. Le gouvernement redécoupe aussitôt les seigneuries démantelées pour les fins d'administration du territoire. Une des recommandations du Rapport Durham ${ }^{66}$, soit celle de créer des municipalités au Canada, se trouve ainsi en voie de réalisation. De 1840 à 1855 s'élabore donc l'actuelle version de la municipalité québécoise. Il s'agit alors de constituer une unification des découpages antérieurs, cette unification ayant principalement pour but d'établir une division du territoire pour d'autres

\footnotetext{
62 Ibid., 140.

63 Nicole Laurin-Frenette, Production de l'État et formes de la nation (Montréal, Nouvelle optique, 1978), 78.

64 Recensements du Canada 1665 à 1871 (Ottawa 1876) 4: 148.

65 Acte pour l'abolition des droits et devoirs féodaux dans le Bas-Canada, 18 décembre 1854, 18 Vict. chap. 3, Statuts du Canada, 1854-1855, 101. 1948), 302 ss.

${ }_{66}$ Le rapport de Durham, présenté, traduit et annoté par M.P. Hamel (Québec,
} 
usages administratifs. La première fonction des municipalités n'a donc pas été l'organisation communautaire, dévolue aux paroisses, mais l'administration de certaines lois, confiées à un niveau local.

Le gouvernement forme un nouveau Comité d'enquête sur l'industrie de la pêche en 1851. Ce comité qui étudie la concurrence des navires de pêche étrangers, remet son rapport l'année suivante $^{67}$. Les quatre principales recommandations du comité couvrent l'ensemble des revendications du secteur de la pêche: l'octroi de primes aux pêcheurs, la construction de phares, la suppression des tarifs sur les agrès, l'organisation d'une police de la mer. La dernière de ces recommandations est bientôt réalisée par le gouvernement, qui crée un Service de protection des pêcheries ${ }^{68}$. Le directeur de ce service, M. Pierre Fortin, devient un allié notoire des compagnies; il sera bientôt élu député. Quelques années plus tard, l'Acte des pêcheries (1858) ${ }^{69}$ rattache ce nouveau service des pêcheries au ministère des Terres de la Couronne. Puis, en 1860, Gaspé devient port franc et le demeure jusqu'en $1866^{70}$. Tous les bateaux de trente tonnes et plus peuvent alors faire admettre en franchise leur cargaison, ce qui donne un essor aux opérations commerciales du port, et amène les pêcheurs à une nouvelle activité d'appoint: la contrebande d'alcool avec les îles françaises Saint-Pierre-et-Miquelon.

\section{A - La Gaspésie après la Confédération}

À la veille de la Confédération, le commerce de la morue atteint son apogée, occupant 5242 pêcheurs par an, environ 1000 marins et 2600 bateaux $^{71}$. Le comté de Gaspé compte alors 14242 habitants ${ }^{72}$.

La signature de la Confédération en 1867 signifie, pour la Gaspésie, une régression importante. Alors que les provinces maritimes font, du développement de leurs ports, la condition de leur entrée dans la Confédération, le Québec se désintéresse complètement du port de Gaspé. La Confédération n'apporte pas non plus les chemins de fer car la Gaspésie est négligée au profit des Mariti-

67 Select Comittee appointed to Inquire into the State of the Fisheries in the Gulf of St-Laurence and the Coast of Labrador, Journals of the Legislative Assembly, 1852, 69.

68 Jules Bélanger et al, Histoire de la Gaspésie, 402-403.

285.

69 Acte des pêcheries, 16 août 1858, 22 Vict. chap. 86, Statuts du Canada, 1858,

70 Rapport des inspections des ponts francs de Gaspé et du Sault Sainte-Marie, Documents de la Session de la province du Canada, XXV, 37 (1865), 54 p.

71 Rapport de Pierre Fortin, dans Rapport du Comité spécial sur le fonctionnement de l'Acte des pêcheries, Journal de la Chambre d'Assemblée de la province du Canada, XXIII, appendice 5: 342 .

72 Recensements du Canada, 1665-1871: 284. 
mes. De plus, avec ce nouveau régime politique, l'espace se trouve de nouveau fortement modifié. En devenant une des provinces canadiennes, le territoire québécois s'agrandit de vastes étendues inoccupées, et les régions habitées vont maintenant s'articuler entre elles en fonction du développement économique.

Le décollage industriel entraîne la mise en place d'une infrastructure de communications, centrée sur Montréal. Déjà bien située pour desservir les marchés ontarien, américain et québécois, Montréal profite d'aménagements d'infrastructure: construction de canaux maritimes et un réseau de chemin de fer.

C'est là que se développent les réseaux de communications et c'est donc à Montréal que les usines s'installent: vêtement, alimentation, textile.

Dans le reste du Québec, au même moment, de nouvelles régions s'ouvrent à la colonisation. Comme remède à la crise économique, l'État et le clergé initient un vaste mouvement de retour à la terre. Une politique de colonisation recrute des colons pour les établir sur de nouvelles terres, de plus en plus éloignées de Montréal et aussi de moins en moins fertiles. L'État fournit l'aide à l'établissement des colons ${ }^{73}$, mais ne se préoccupe pas des réseaux de communication. De 1850 à 1900 , les nouveaux arrivants occupent les plateaux et commencent lentement à peupler le Témiscamingue et le Saguenay-Lac Saint-Jean. Ces grandes opérations de colonisation, jointes à l'industrialisation de la région de Montréal, signifient pour la Gaspésie un accroissement de l'écart qui la sépare des autres régions: l'accroissement de ses inégalités de peuplement et de ses disparités régionales.

Les difficultés de communication et l'éloignement empêchent en effet la Gaspésie de devenir une véritable région de colonisation. La pêche demeure la principale ressource régionale, et la péninsule se développe selon une perspective d'activités complémentaires. L'État encourage l'agriculture; les agents des terres distribueront en vingt ans (1867-1887) 206 billets de concession dans le comté de Gaspé ${ }^{74}$. Mais la faiblesse des marchés, le peu de rendement et la faible superficie des terres limitent l'agriculture gaspésienne à la culture autarcique de la pomme de terre. L'agriculture,

73 En vertu de la Loi des terres de colonisation (Statuts révisés du Québec, 1964, chap. 102) les colons reçoivent un billet de location pour leur terre. Une fois le lot défriché, aménagé et bâti, le colon peut demander des lettres patentes et obtenir ainsi un titre de propriété. La Loi de la protection des colons (Statuts révisés du Québec, 1964, chap. 107) exempte les colons de toutes saisies pendant six ans. La Loi du mérite du défricheur 1964 (Statuts révisés du Québec, chap. 107) récompense le défrichement et la contribution à l'oeuvre de la colonisation (art. 2).

${ }_{74}$ Jules Bélanger et al, Histoire de la Gaspésie, 320 ss. 
marginale, pousse les habitants à cumuler les ressources et les emplois; ils doivent pêcher ou bûcher pour survivre. Par ailleurs, comme l'agriculture est subventionnée, les salaires versés par les compagnies restent très en deçà des salaires urbains. Le «mode de vie» gaspésien s'établit ainsi entre deux activités subventionnées, la pêche et l'agriculture, et une troisième activité, le travail forestier, saisonnier et sous-payé.

À la veille de la Confédération, le gouvernement du BasCanada subventionne déjà les pêcheries gaspésiennes. Le Service des pêcheries rapporte pour les années 1860, 1861 et 1862 des dépenses de 29090 dollars, 23471 dollars et 28392 dollars respectivement, sommes qui incluent les salaires et les subventions ${ }^{75}$. Ces subventions doivent être demandées au Service des pêcheries et à la Chambre d'Assemblée par pétitions, l'État n'accordant aucune aide statutaire à la région. L'indifférence des autorités civiles, l'oubli de la province et du Canada, la mainmise des compagnies de bois et de pêche caractérisent encore le XIXe siècle gaspésien ${ }^{76}$. L'isolement de la région se compare en tous points à celui des siècles précédents.

À partir de 1867, un seul député représente le comté de Gaspé au Parlement fédéral et à l'Assemblée nationale: Pierre Fortin, le directeur du Service des pêcheries. Ce dernier cumule les deux mandats de député tant que ce cumul demeure possible, c'est-àdire jusqu'en $1886^{77}$.

Dans le partage des pouvoirs établi par la Confédération, les pêcheries ressortissent aux deux juridictions ${ }^{78}$. Dès lors, les subventions et les législations proviennent des deux paliers de gouvernement. L'État provincial détient les droits de propriété et de juridiction sur toutes les eaux, sauf dans les havres publics, lesquels deviennent de juridiction fédérale ${ }^{79}$. Le gouvernement du Québec continue alors la politique du Bas-Canada et concède des droits de pêche exclusifs jusqu'à l'adoption de sa première loi sur les pêcheries en 1883. Â partir de ce moment, l'État ne peut plus aliéner ces droits à perpétuité et les baux n'excèdent pas 9 à 15 ans ${ }^{80}$. La loi de 1883 traite des droits de pêche, des baux et permis, des circonscriptions et des pouvoirs des garde-pêche ${ }^{81}$.

75 Rapport du Comité spécial sur le fonctionnement de l'Acte des pêcheries, Journaux de la Chambre d'Assemblée, 1864, XXIII: appendice 5.

76 Antoine Bernard, La Gaspésie au soleil, précité, 202.

77 Le cumul de mandats devient impossible en 1886, An Act respective The House of Commons, Statuts refondus du Canada, 1886, c.13, art. 1: 191 .

${ }_{78}$ Art. 91 (12) et 92 (10) de l'Acte de l'Amérique du Nord britannique.

79 The Queen c Robertson, Cour Suprême du Canada, 1897, Cartwright, 2: 165.

8046 Vict. chap. 8, 1883; Statuts refondus du Québec, 1888, art. 1376.

81 Ibid., art. 1374 à 1389. 
Durant les années 1880 , le gouvernement fédéral adopte aussi ses propres lois concernant les pêcheries: la Loi sur les pêcheries ${ }^{82}$, la Loi relative aux pêcheries par des bateaux étrangers ${ }^{83}$ et la $L o i$ pour encourager le développement des pêcheries maritimes ${ }^{84}$. Cette dernière loi contient des dispositions qui permettent aux ministres d'accorder des subventions annuelles pour un montant de 150000 dollars $^{85}$. Â partir de 1882 , le gouvernement fédéral distribue ainsi des primes aux «pêcheurs méritants». Pour obtenir cette prime annuelle de cinq dollars, le pêcheur doit posséder un bateau de pêche de 12 pieds et plus, s'adonner à la pêche au moins trois mois par année et pêcher un minimum de 2500 livres de poisson. Ces primes sont accordées après une déclaration assermentée des pêcheurs ${ }^{86}$. À l'exception de ces primes, les subventions fédérales vont aux compagnies de construction navale et financent la police des mers. Un peu plus tard, le gouvernement fédéral adopte une Loi concernant les douanes et les pêcheries ${ }^{87}$.

Avec la Confédération, les pêcheries deviennent donc un domaine de juridiction partagée, et les législations se chevauchent. $\mathrm{Au}$ cours des années, la juridiction partagée sur les pêcheries donne lieu à un conflit fédéral-provincial auquel l'arbitrage du Conseil privé de Londres apporte une solution mitoyenne: le fédéral conserve son pouvoir législatif, mais les inspecteurs, désormais nommés et payés par la province, appliquent les lois fédérales ${ }^{88}$. Après 1922, le gouvernement fédéral n'assume plus l'entretien des pêcheries québécoises. Le Québec administre ses pêcheries et le Canada continue de décider des saisons de pêche, de la nature et de la forme des engins de capture, de la classification des produits et de leur mise en conserve.

\section{$B$ - La crise de la morue}

La pêche à la morue, demeurée artisanale, accuse alors un retard technologique et commercial important à la fin du XIXe siècle. Puis, au début du XXe siècle, c'est la crise. Le développement

\footnotetext{
82 An Act respecting Fisheries and Fishing, Statuts refondus du Canada, 1886, chap. $95,1257$.

${ }_{83}$ An Act respecting Fishing by Foreing Vessels, Statuts refondus du Canada, 1886, chap. 94, 1251.

${ }_{84}$ An Act to Encourage the development of sea fisheries and the building of fishing vessels, Statuts refondus du Canada, 1886, chap. 96, 1273.

85 Ibid., art. 2.

${ }^{86}$ Firmin Létourneau, "La Côte Nord de Gaspé», Revue d'histoire de la Gaspésie, III, 4 (octobre-décembre 1965): 42. chap. 47 .

87 An Act to protect the Customs and Fisheries, Statuts refondus du Canada, 1909,

${ }_{88}$ Attorney General for Canada c. Attorney General for the Province of Québec, Decisions of the Judicial Committee of the Privy Council, Olmsted, 2: 174.
} 
des transports et des communications fait peu à peu disparaître la flotte des compagnies, et amène la désaffectation des petits ports. Le nombre de pêcheurs diminue. Un pêcheur qui peut alors prendre jusqu'à 70 quintaux de 112 livres de morue par saison, reçoit de trois à cinq dollars par quintal ${ }^{89}$. En 1908, à Rivière-au-Renard, les compagnies annoncent qu'elles paieront cinq dollars le quintal. Quelques semaines plus tard, ce prix est fixé à trois dollars. Les pêcheurs en colère envoient une délégation aux compagnies pour réclamer quatre dollars le quintal. Un pêcheur est blessé par les agents des compagnies, qui sont à leur tour battus par les pêcheurs. Puis chacun rentre chez soi après cette mêlée qui reçoit le nom de «Révolte des pêcheurs». Le gouvernement fédéral envoie à la rescousse des compagnies une frégate remplie de soldats, qui fouillent les maisons, arrêtent des pêcheurs et font régner la terreur pendant plus d'une semaine. Au terme d'un procès, vingt pêcheurs seront emprisonnés à Percé ${ }^{90}$. Cette «Révolte» est suivie en 1909 de trois législations: la Loi de l'engagement des pêcheurs ${ }^{91}$, prévoyant des amendes de 40 dollars pour bris d'engagement et désertion, la Loi du Service des pêcheries maritimes ${ }^{92}$, favorisant la pisciculture et la création d'associations coopératives de pêcheurs et la Loi concernant les associations de pêcheurs pour l'exploitation de la boette 93 .

Quelques années plus tard, une brèche s'ouvre dans la grande solitude gaspésienne. En 1913, un premier train de passagers entre en gare de Gaspé. Puis en 1929, on entreprend la construction de la première route de ceinture, le long du littoral. Le réseau routier contribue ainsi à rompre l'isolement de la péninsule. Il faut cependant attendre la fin des années quarante pour que l'avion, le téléphone et l'électricité rompent définitivement cet isolement géographique, transformant dès lors la Gaspésie en «région éloignée», articulée davantage au reste du Québec.

Pour la Gaspésie, les années de crise économique suivent les années difficiles de la pêche à la morue. Le clergé encourage toujours l'agriculture ${ }^{94}$. Un évêque, Mgr Ross, contribue grandement à la colonisation de la Gaspésie, favorise la construction d'institutions à Gaspé, développant et francisant la ville. À la fin des années trente, la région, toujours isolée et oubliée, suscite l'inquiétude des autorités civiles, tant elle échappe à la connaissance

\footnotetext{
89 Firmin Létourneau, «La Côte Nord de Gaspé», précité, 41-42.

90 Augustine Jalbert-Côté, «La révolte de 1909 à Rivière-au-Renard», Revue d'histoire de la Gaspésie, IV, 1 (janvier-mars 1966): 5-9.

91 Statuts refondus de la province de Québec, 1925, chap. 273.

92 Statuts refondus de la province de Québec, 1925, chap. 84.

93 Statuts refondus de la province de Québec, 1925, chap. 85.

94 Antoine Bernard, La Gaspésie au Soleil, précité, 270 ss.
} 
sociale. C'est alors le début d'une série d'enquêtes étatiques, qui précèdent les premiers programmes d'aménagement du territoire.

Le gouvernement Duplessis s'inquiète de la situation économique de la province et mène, dès 1937, des recherches sur les ressources de chacun des comtés. L'enquête commence en Gaspésie, sous la direction de l'économiste Esdras Minville ${ }^{95}$. Cette étude révèle que les statistiques officielles des pêcheries multiplient régulièrement les données réelles par trois. Ainsi les enquêteurs dénombrent 680 barques, alors que les statistiques gouvernementales mentionnent 1940 barques. Cette étude révèle aussi que les pêcheurs gagnent tout au plus deux cent cinquante dollars par an, en vendant cinquante mille livres de poisson à un cent et demi la livre. Quant au revenu moyen, il s'élève en 1937 à 161 dollars par famille par année. On attribue ces bas revenus aux caprices de la pêche, mais aussi aux prises massives des chalutiers, qui s'approchent encore plus près des rives que les limites de douze milles marins le permettent. L'enquête démontre encore que la production agricole est insuffisante pour couvrir les besoins familiaux, car elle se pratique à une échelle limitée dans l'espace et le temps. À certains endroits, tels Cloridorme ou Saint-Octave-de-l'Avenir, la colonisation s'est faite sur des terres tellement rocheuses que rien ne peut y être cultivé. La forêt apparaît alors comme un revenu d'appoint essentiel, et les Gaspésiens s'habituent au travail mixte. Cependant, sur quatre-vingt-treize moulins à scie, trentedeux manquent de bois car les forêts sont concédées aux grandes compagnies forestières: une Compagnie détient à elle seule quatre blocs de mille milles carrés chacun ${ }^{96}$. Quant aux mines et au tourisme, l'état des routes n'en favorise pas le développement. L'agriculture représente la principale ressource de soixante-quinze pour cent de la population. On cultive le foin, l'avoine et la pomme de terre, dans des exploitations relativement petites où le capital investi ne dépasse guère deux mille dollars. L'enquête Minville conclut en énumérant quatre causes du retard gaspésien: le blocage du domaine public par les seigneuries et les concessions forestières, le manque de coordination des différentes ressources, l'insuffisance des communications et l'inadaptation du régime économique ${ }^{97}$.

95 Jules Bélanger, et al, Histoire de la Gaspésie, précité, 544.

96 Joseph Risi, «Petite histoire d'une vieille étude économique en Gaspésie», dans La problématique du développement en milieu rural, Actes du colloque de l'UQAR (Rimouski, Groupe de recherche et d'intervention sur le développement de l'Est du QuébecUQAR, 1976), 201.

97 Ibid., 202. 
L'enquête Minville révèle des conséquences du chômage à ce point graves que le gouvernement adopte la Loi pour promouvoir le développement de la Gaspésie ${ }^{98}$, appelant des soumissions pour la réouverture de l'usine de pâtes et papier de Chandler. L'État subventionne aussi la construction d'entrepôts frigorifiques avec la Loi favorisant le progrès et venant en aide aux pêcheurs ${ }^{99}$. Une troisième législation encourage la prospection: la Loi relative aux ressources pétrolières de la Gaspésie ${ }^{100}$. Le gouvernement y établit en même temps un relais touristique, aménage un parc public de récréation dans la péninsule: le parc national de la Gaspésie ${ }^{101}$. Ce parc, qui couvre alors une étendue de 340 milles carrés ${ }^{102}$, comptera en 1960 plus de 1300 milles carrés. L'État crée en même temps l'Office du tourisme, pour promouvoir ces nouveaux aménagements à l'étranger ${ }^{103}$. Mais la misère des Gaspésiens n'est en rien allégée.

Huit ans après Esdras Minville, en 1944, l'historien Robert Rumilly reprend la même enquête, pour attirer l'attention de l'opinion publique ${ }^{104}$. La Gaspésie n'est pas encore électrifiée, le téléphone n'y est installé, à certains endroits, que depuis la guerre et les communications par terre sont bien pénibles. Il n'y a pas de route intérieure.

En 1951, la région de l'Est du Québec compte 221053 habitants, soit $5,4 \%$ de la population québécoise. Le comté de Gaspé compte 62530 habitants ${ }^{105}$. Dix ans plus tard, en 1961, la population aura diminué à 61872 habitants ${ }^{106}$. L'exode des Gaspésiens, initié pendant les années trente, se poursuit à un rythme affolant. Les pêcheries connaissent la plus importante crise depuis le début du siècle. Cette crise affecte surtout les petits pêcheurs et l'organisation économique des villages. Dans un village de la baie des Chaleurs, en 1953, sur 233 individus susceptibles de pêcher, seuls 33 pratiquent ce métier; de ce nombre, 10 abandonnent durant la saison, 12 pêchent en haute mer sur des chalutiers, et 11 seulement pratiquent la pêche traditionnelle ${ }^{107}$. Les jeunes quittent leur village pour travailler dans les mines et les camps de bûcherons du Nord. La morue, qui se vendait un cent et quart la livre en 1912, se

\footnotetext{
98 Statuts du Québec, 1936, chap. 23.

99 Statuts du Québec, 1937, chap. 46.

100 Statuts du Québec, 1936, chap. 22.

101 Statuts du Québec, 1937, chap. 47.

102 a.c. 2397, 14 septembre 1937, Gazette officielle du Québec, no 69, 3672.

103 Statuts du Québec, 1937, chap. 48.

104 Robert Rumilly, La Gaspésie, Enquête économique, Québec, Le Soleil, 1944.

105 Recensement du Canada, 1951, vol. 1, tableau 7.

106 Recensement du Canada, 1961, vol. 1, tableau 7.

107 Marcel Rioux, Belle-Anse, Musée national du Canada (Imprimeur de la reine, Ottawa, 1957), 20.
} 
vend deux cents et demi en 1953. Et cette somme est largement subventionnée: les gouvernements assument quatre-vingt-deux cents pour chaque dollar gagné par les pêcheurs ${ }^{108}$.

L'intérêt des habitants s'éveille aux questions politiques en relation avec l'aide économique qu'ils peuvent en retirer: c'est-àdire des emplois, de l'aide sociale, la construction des quais, des édifices publics, l'aide aux entreprises. Du gouvernement fédéral, les villages attendent la construction des bureaux de poste et des quais, et reçoivent régulièrement des prestations. Ils n'ont pas à quémander les services sociaux fédéraux; allocations familiales, pensions de vieillesse, assurance-chômage sont des services statutaires ${ }^{109}$. Les pêcheurs deviennent d'ailleurs éligibles à l'assurancechômage en $1957^{110}$. L'obtention de l'aide provinciale réclame au contraire de multiples démarches. Les octrois provinciaux ne sont jamais assurés, ni leur renouvellement, ni le maintien des services sociaux. Tout dépend du député, tout dépend du Parti au pouvoir. Aussi les élections provinciales en Gaspésie tiennent-elles plus de place que les élections fédérales ${ }^{111}$.

\section{C - Les programmes d'aménagement du territoire}

L'intervention ponctuelle du gouvernement provincial en Gaspésie s'appuie sur de nombreux rapports de Commissions. Le rapport Héon ${ }^{112}$, en 1955, suivi d'une modification de la Loi des marchés agricoles ${ }^{113}$, favorise la conclusion de plans conjoints pour la mise en marché de produits agricoles et du bois, entre acheteurs et petits producteurs. Un premier plan est bientôt approuvé pour la Gaspésie: le plan conjoint des producteurs de bois de pulpe de la Gaspésie, ratifié par $75 \%$ des producteurs ${ }^{114}$.

C'est toutefois la Commission d'enquête sur les problèmes constitutionnels ${ }^{115}$ qui déclenche l'intervention concertée. Cette

108 G.-E. Blanchard, La Gaspésie, ordonnance de ses valeurs et problèmes constitutionnels, précité.

${ }_{109}$ Loi sur les allocations familiales, Statuts refondus du Canada, 1952, V. II: chap. 109. Loi sur l'assurance-chômage, Statuts révisés du Canada, 1952, V. IV: c. 273. Loi sur les pensions de vieillesse, Statuts révisés du Canada 1952, V. III: c. 199.

110 C.P. 1957-442, 28 mars 1957, DORS 57-116, Gazette officielle du Canada, 1957, partie II: 333.

111 Marcel Rioux, Belle-Anse, précité, 42.

112 Rapport du comité d'enquête pour la protection des agriculteurs et des consommateurs (Commission Héon, a.c. 9 avril 1952) (Québec, 1955), 375 ss.

113 Loi des marchés agricoles, Statuts refondus du Québec, 1964, c. 120, art 20 à 26.

11421 mai 1958, 47878-0, Gazette officielle du Québec, 1958, 1961.

115 Loi instituant une Commission d'enquête sur les problemes constitutionnels, 12 février 1953, Statuts du Québec, 1953, c. 4. 
Commission d'enquête remet son rapport en $1956^{116}$; ce rapport constitue un plaidoyer pour l'autonomie provinciale et une déclaration d'intention de la part du gouvernement de prendre en main la situation québécoise. À l'appui des conclusions du rapport: une enquête sur la situation régionale. En Gaspésie, la Commission constate que la situation économique et sociale n'a guère changé depuis le rapport Minville. La dispersion de la population, de même que la non-disponibilité et l'exploitation des ressources naturelles ont créé une situation de déséquilibre chronique.

Ce déséquilibre se fonde sur l'exploitation de deux ressources principales: les pêcheries et le tourisme. Les forêts gaspésiennes, contrairement aux forêts du bas Saint-Laurent, ne constituent pas une importante ressource; pillées, elles ont vite été presque épuisées. Dans les années cinquante, le tourisme démarre. Des installations touristiques se construisent le long du littoral. En 1956, cent mille visiteurs font le tour de la Gaspésie, dépensant 4 millions de dollars dans la péninsule ${ }^{117}$. Dans son mémoire à la Commission Tremblay, le porte-parole des groupes gaspésiens souhaite que le tourisme profite d'une particularité de l'été gaspésien, lequel se prolonge durant le mois de septembre ${ }^{118}$. Les Gaspésiens souhaitent aussi que le gouvernement contribue à intensifier l'agriculture et fasse cesser le pillage des forêts. À cette époque la Gaspésie se compose surtout de municipalités rurales $(98 \%)$, dont la population moyenne atteint 1250 habitants. Aucune municipalité ne compte plus de 4000 habitants.

Depuis que la Gaspésie a commencé à se peupler, les gouvernements ont favorisé l'exploitation des pêcheries. L'exploitation du tourisme se fait selon le même modèle: le capital vient de l'extérieur de la région, et le développement du tourisme appauvrit davantage la population et les ressources régionales. Le tourisme favorise surtout l'État, qui perçoit jusqu'à $25 \%$ des recettes, et les investisseurs, petits, moyens ou gros. La création d'emplois locaux et non spécialisés demeure marginale. Selon une étude de l'UNESCO, les plus démunis sont de nouveau lésés par le développement du tourisme: l'éloignement des pêcheurs de leur maison et du marché, l'interdiction de la chasse dans les parcs nationaux, l'élimination des témoignages de pauvreté, voilà autant d'effets immédiats de l'exploitation du tourisme ${ }^{119}$.

${ }^{116}$ Rapport de la Commission royale d'enquête sur les problèmes constitutionnels, (Rapport Tremblay), 4 volumes, 11 annexes (Québec 1956). Voir le Volume III, sur la Gaspésie et l'annexe no 7: Albert Rioux, Le problème rural, 1955, 42ss.

117 Jules Bélanger, et al, Histoire de la Gaspésie, précité, 618.

118 G.-E. Blanchard, La Gaspésie, ordonnance de ses valeurs..., précité.

119 Emmanuel de Kadt, Tourisme, passeport pour le développement (Unesco, Paris, Economica, 1979), 45. 
Les premiers efforts d'aménagement ont donc eu pour conséquence immédiate d'accélérer les départs et les fermetures. De 1951 à 1961, l'Est du Québec perd 8700 fermes, malgré les programmes gouvernementaux d'aide à l'agriculture ${ }^{120}$. Au début des années soixante, la Gaspésie représente une priorité pour l'État. Le nouvel État de la Révolution tranquille y voit la région la plus défavorisée du Québec, et partant, la première à aménager. Cette fois encore, l'État procède d'abord par une enquête. Le Bureau d'Aménagement de l'Est du Québec, formé en 1963, remet son volumineux rapport en 1966. Les recommandations concernant l'économie reprennent les mêmes thèmes: développer davantage le secteur des pêcheries et le tourisme en Gaspésie.

$\mathrm{Au}$ début des années soixante, l'État devient omniprésent en Gaspésie. À cette époque, $60 \%$ de la population de la péninsule vit de prestations d'aide sociale et d'assurance-chômage ${ }^{121}$. Les pêcheries aussi dépendent des gouvernements: le ministère de l'Industrie et du Commerce du Québec assume $25 \%$ du coût de construction des bateaux de pêche, et contribue au paiement des intérêts et des assurances sur ces bateaux; le gouvernement provincial accorde également des rabais sur l'essence utilisée par les pêcheurs et construit des entrepôts frigorifiques pour le poisson. Quant au gouvernement fédéral, il subventionne à son tour la construction des bateaux de pêche ( 25 à $40 \%$ selon le tonnage), assume une partie des assurances sur ces bateaux et fournit $50 \%$ du sel ${ }^{122}$.

Depuis les années 1840, l'État remplace peu à peu les compagnies de pêche comme "gouverneur» de la Gaspésie. L'État s'insère d'abord comme intermédiaire entre les compagnies et les Gaspésiens, arbitrant leurs différends et légiférant dans le sens des intérêts des compagnies. Puis l'État devient lui-même producteur de services en aménageant des installations et en subventionnant directement les pêcheurs et les compagnies de pêche. La situation féodale de la Gaspésie ne change pas, mais les autorités gouvernementales se substituent aux compagnies jersiaises, lesquelles ne parviennent plus à assumer seules le contrôle social.

$\mathrm{Au}$ début des années soixante, la région devient tellement dépendante de l'État que les gouvernements en sont totalement maîtres. Ce contrôle va permettre aux gouvernements d'initier en

120 Pierre Dufort, «Comment sous-développer rationnellement l'Est du Québec, ou l'aménagement vu par les aménagés» dans La problématique du développement en milieu rural, Actes du Colloque de l'UQAR, 24-25 octobre 1975 (Rimouski, GRIDEQ-UQAR, 1976).

121 G.-E. Blanchard, La Gaspésie, ordonnance de ses valeurs..., précité. précité.

122 Firmin Létourneau, «La Côte-Nord de Gaspé», Revue historique de la Gaspésie, 
Gaspésie un vaste programme de restructuration spatiale. Selon les gouvernements, pour que les pêcheries demeurent concurrentielles et le tourisme devienne rentable, la Gaspésie doit se moderniser. Aux dires des gouvernements, l'absence de développement des ressources gaspésiennes doit être attribuée aux disparités régionales et à une structure économique trop traditionnelle. La solution devient donc de moderniser et d'urbaniser la Gaspésie, pour exploiter de façon plus rationnelle les pêcheries et le tourisme.

Pour réaliser ces objectifs, à la suite du rapport du BAEQ, les gouvernements concluent des ententes fédérales-provinciales de développement en Gaspésie. Ces programmes spéciaux n'ont pas pour conséquence le développement de ressources nouvelles, mais bien la «modernisation» du secteur des pêcheries et du tourisme. Pendant vingt ans, le territoire de la péninsule sera complètement remodelé, refaçonné, pour satisfaire aux nouvelles exigences concernant l'exploitation rationnelle des pêcheries et du tourisme.

Encore une fois, le développement de la Gaspésie s'oriente vers les mêmes avenues: les pêcheries et le tourisme ${ }^{123}$.

\section{CONCLUSION}

Il s'agit là d'une continuité. Le peuplement et le quasidéveloppement de la Gaspésie originent de la volonté d'exploiter les pêcheries du golfe Saint-Laurent. Ces ressources intéressent d'abord les compagnies jersiaises, dont l'État concourt à assurer la prospérité jusqu'au milieu du XIXe siècle. La Compagnie Robin peut ainsi édifier en Gaspésie un véritable empire.

À partir des années 1840, quand l'État canadien et québécois se constitue, il prend peu à peu le contrôle de l'industrie de la pêche, participant de plus en plus directement à l'exploitation des pêcheries du golfe. Cette industrie continue d'échapper aux Gaspésiens qui n'obtiennent pas leur part de profit. Les ressources de la mer sont encore exploitées par des compagnies situées à l'extérieur de la région, qui ne recrutent sur place qu'une main-d'oeuvre bon marché. Cent ans plus tard, la population gaspésienne se trouve sous un contrôle étatique presque total, subsistant grâce aux paiements de transfert et aux subventions gouvernementales aux pêcheries. La re-territorialisation de la Gaspésie des années 1960 ne change pas les orientations du développement: l'État méconnaît encore l'exploitation des pêcheries et du tourisme. Pêcheries et tourisme deviennent les deux volets d'un développement axé sur l'exploitation d'une seule ressource, la mer.

123 OPDQ, Les orientations économiques de l'Est du Québec, (Québec, 1979); OPDQ, L'Est du Québec d'hier à aujourd'hui (Québec, 1980). 
La modernisation de la Gaspésie change cependant la représentation de ce territoire à l'échelle provinciale. Cette restructuration change aussi l'articulation de cette région à l'ensemble des régions québécoises. De région isolée, oubliée, la Gaspésie passe à une représentation comme "région éloignée», greffée à l'ensemble du territoire québécois. La Gaspésie acquiert ainsi un statut de complémentarité, en fait un statut de parent pauvre. C'est une «région éloignée» dont le développement ne peut égaler celui des villes moyennes. L'éloignement justifie alors en partie le nondéveloppement des ressources locales.

En aménageant la région, l'État rompt l'isolement, mais il ne crée qu'une seule communication: de l'Etat vers la région, de la région vers l'État. La nouvelle articulation de la Gaspésie à l'ensemble du Québec est l'oeuvre de l'État. La plupart des échanges extra-régionaux se font maintenant entre services de l'État: d'un bureau sous-régional à un bureau régional, puis au bureau central. La «région éloignée» gravite ainsi autour du Centre-État, qui la crée, l'aménage et en perpétue les difficultés.

Au cours des années, l'État s'est substitué aux compagnies de pêche pour aménager directement cette dépendance, garante d'un contrôle social effectué à distance. La Gaspésie, région éloignée, apparaît depuis toujours vouée à la seule exploitation des ressources de la mer. Les grands programmes d'aménagement des années soixante n'opèrent pas de changement dans cette structure économique, mais resserrent le contrôle de l'État sur les activités régionales. Ainsi, la Gaspésie se retrouve encore dans cette situation de dépendance, une dépendance instituée, entretenue et reproduite par l'État. 